\title{
Don't trust your intuition
}

Several years ago, researchers gave bright college students at Princeton University and the University of Michigan the following simple problem. A bat and ball together cost $\$ 1.10$. The bat costs $\$ 1$ more than the ball. How much is the ball? Roughly half the students at both universities gave the wrong answer. They said the ball costs 10 cents, rather that the correct 5 cents.

Almost everyone has an initial tendency to give the same 10-cent answer, a tendency illustrating the potential for intuition - as a kind of instinctive thinking - to defeat our more rational mind. The intuitive mind works effortlessly, sees that $\$ 1.10$ splits easily into $\$ 1$ and 10 cents, which differ by roughly the right amount, and spits out an answer. Rational thinking takes effort.

The same two minds also do battle within every physicist. Intuitively, for example, we feel that particles with like charges should repel one another, even though plenty of examples show exceptions to the rule.

The DNA double-helix carries a negative charge of roughly $-e$ per $1.7 \mathrm{~nm}$ of length. In a weak solution with free positive ions, these negative charges are partially screened out, but they still generally repel one another. No surprise there. But funny things happen if you increase the concentration of ions, and if each carries a multiple charge of, say, $+4 e$. At some point, the DNA molecules suddenly begin to attract one another and collapse together in a clump. At higher concentrations still, the weird attraction goes away and DNA again disperses within the solution. What's going on?

The secret, it seems, is 'charge inversion' - a surprising phenomenon in which a strongly charged particle can attract so many surrounding multiply charged ions that it becomes 'over-screened' and its charge effectively reversed. That surely seems non-intuitive, but it actually makes perfect rational sense.

Think of a charged DNA molecule that has already attracted screening ions to its surface. If these ions don't screen the DNA charge completely, it will still attract others, if they are available, until they neutralize the DNA complex. But that's not all. Further positive charges will still be attracted, by the same subtle physics that can pull a charge towards a neutral metallic particle. By polarizing the distribution of charge on the DNA surface - which effectively form a strongly correlated

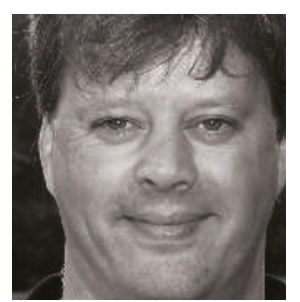

liquid on that surface - the charge creates a negative image to which it is then attracted.

Overcharging has been observed clearly in experiments, where it alters the direction of a particle's drift in an electric field. And it seems to explain DNA 'clumping' too. At low positive-ion concentrations,

ELECTROSTATIC 'OVER-

SCREENING'

SEEMS NON-

INTUITIVE, BUT

IT ACTUALLY

MAKES PERFECT

RATIONAL SENSE. the DNA molecules repel because they're not fully screened and effectively negative. At high concentrations, they're over-screened and effectively positive, with the same result. Only at a critical concentration of screening charges do the DNA molecules become effectively neutral - and they then attract one another by weaker short-range forces.

It's likely that biology puts this non-intuitive physics to good use, in packing DNA tightly within chromosomes, for example (for a review, see A. Grosberg et al. Rev. Mod. Phys. 74, 329-345; 2002). Of course, electrons sometimes bind together in Cooper pairs. Two electrons can be bound together by a single proton, as in the hydrogen negative ion. There's nothing really surprising here; it's just that our intuition for electrostatics - or mine, at least — still has some lessons to learn.

Mark Buchanan

\section{What's in a theory?}

I know I am going to get into trouble here. Each time I write about string theory I manage to offend some young researchers in that field. On a popular physics blog (http://cosmicvariance. com), having been asked to defend my views, I raised an issue that prompted some thoughtful discussion, so I present it here for a broader audience.

There is no denying that the string enterprise involves an impressive body of theoretical work. However, it is equally clear that what we normally call string theory is not a 'theory' in the conventional scientific sense. String theory does not yet make sharp quantitative assertions about specific phenomena (as electroweak theory does), nor does it make falsifiable qualitative predictions about observable phenomena (as evolutionary theory does). The term 'string theory' is in fact a historical anachronism, created to distinguish it from another formalism developed to deal with the relativistic quantum mechanics of point particles - field theory.

This would be mere nitpicking except for the fact that, as I described in an earlier column, we are currently fighting a battle against those who wish to water down the science we teach in high schools. Part of the argument of those pushing intelligent design is that evolution is "just a theory", referring to the common parlance and not the more restrictive sense in which the term is usually discussed in science.
Labelling as theories ideas that have yet to pass the necessary empirical tests or develop a highly constrained logical formalism - strings in particle physics, or inflation in cosmology - opens us up to otherwise avoidable attacks, particularly from those who would include religious ideas in highschool science curricula.

If, instead, we train ourselves to refer to ideas such as strings and inflation as hypotheses or paradigms - just as Eugenie Scott of the US National Center for Science Education has said, we should avoid the term 'believe' in a scientific context - we might save ourselves grief down the line. Even if I might get grief now for suggesting it.

Lawrence M. Krauss 